\title{
ON THE RANGE OF A HOMOMORPHISM OF A GROUP ALGEBRA INTO A MEASURE ALGEBRA
}

\author{
JYUNJI INOUE
}

\begin{abstract}
It is shown, that if $G$ is a LCA group and if $H$ is a nondiscrete LCA group then there exists a proper closed subalgebra of the measure algebra of $H$ (independent of the choice of $G$ ) in which the range of every homomorphism of the group algebra of $G$ into the measure algebra of $H$ is contained.
\end{abstract}

Throughout this paper, $G$ and $H$ denote LCA groups and $\hat{G}$ and $\hat{H}$ denote their dual groups, respectively. $\mathfrak{I}(H)$ is the set of all the locally compact group topologies of $H$ which are at least as strong as the original one of $H$. For each $\tau \in \mathfrak{I}(H)$, if we denote by $H^{r}$ a LCA group with underlying group $H$ and topology $\tau$, the natural continuous isomorphism of $H^{\tau}$ onto $H, x \in H^{\top} \mapsto x \in H$, induces a natural norm-preserving imbedding of $L^{1}\left(H^{\top}\right)$ into $M(H)$, which we also denote by $L^{1}\left(H^{\top}\right)$. For the other notations and terminologies which we need in this paper, we follow [6].

The author would like to express his thanks to the referee. His kind advice enabled the author to make this paper more readable.

THEOREM. If $h$ is a homomorphism of $L^{1}(G)$ into $M(H)$, then there exist finitely many elements $\tau_{1}, \tau_{2}, \cdots, \tau_{n} \in \mathfrak{I}(H)$ such that the range of $h$ is contained in $\sum_{i=1}^{n} L^{1}\left(H^{\tau_{i}}\right)$.

For the proof of the theorem, we essentially use Cohen's results, which determine all the homomorphisms of $L^{1}(G)$ into $M(H)$ by the notion of the coset ring and piecewise affine maps (cf. [1], [2], [3] and [6, Chapters 3 and 4]).

If $h$ is a homomorphism of $L^{1}(G)$ into $M(H)$, Cohen's theorem asserts that there exist $Y$, an element of the coset ring of $\hat{H}$, and a piecewise affine map $\alpha$ from $Y$ into $\hat{G}$ such that

$$
\begin{aligned}
h(f)^{\wedge}(r) & =\hat{f}(\alpha(r)), & & r \in Y \\
& =0, & & r \notin Y
\end{aligned} \quad\left(f \in L^{1}(G)\right),
$$

Received by the editors August 2, 1971 and, in revised form, June 18, 1973. AMS (MOS) subject classifications (1970). Primary 43A22, 43A10.

Key words and phrases. Homomorphisms of group algebras, measure algebras, LCA groups, range of homomorphisms.

(c) American Mathematical Society 1974 
and conversely, if $Y$ is an element of the coset ring of $\hat{H}$ and if $\alpha$ is a piecewise affine map from $Y$ into $\hat{G}$, the pair $(Y, \alpha)$ induces a unique homomorphism $h$ of $L^{1}(G)$ into $M(H)$ which satisfies (1). We call the pair $(Y, \alpha)$ the dual map of $h$ after P. Eymard [3] (though slightly different from his definition).

For the rest of this paper, $h$ denotes a homomorphism of $L^{1}(G)$ into $M(H)$ and $(Y, \alpha)$ denotes the dual map of $h$.

LEMMA 1. If $Y$ is an open subgroup of $\hat{H}$ and $\alpha$ is a continuous homomorphism from $Y$ into $\hat{G}$, then the range of $h$ is contained in $L^{1}\left(H^{\top}\right)$ for some $\tau \in \mathfrak{I}(H)$.

Proof. We suppose first that $Y=\hat{H}$ and $\alpha(Y)$ is dense in $\hat{G}$, and then there exists a natural continuous isomorphism $\hat{\alpha}$ of $G$ into $H$ such that

$$
(\hat{\alpha}(x), r)=(x, \alpha(r)) \quad(x \in G, r \in \hat{H}) .
$$

We can introduce in $H$ a locally compact group topology $\tau$ such that $\hat{\alpha}$ becomes an open continuous map of $G$ into $H^{\tau}$, and then $\hat{\alpha}$ induces the natural isomorphism of $L^{1}(G)$ into $L^{1}\left(H^{r}\right)$, which just coincides with $h$.

Next we suppose only that $\alpha(Y)$ is dense in $\hat{G}$. By the above considerations, we have an element $\bar{\tau} \in \mathfrak{I}(H / L)$ and a continuous isomorphism $h$ of $L^{1}(G)$ into $L^{1}\left((H / L)^{\tilde{\tau}}\right)$ such that the dual map of $\bar{h}$ is $(Y, \alpha)$, where $L$ denotes the annihilator of $Y$ in $H$.

Let $\pi$ be the natural map of $H$ onto $H / L$. If we introduce in $H$ a topology $\tau$ with a basis $\left[\pi^{-1}(V) \cap W: V\right.$ is open in $(H / L)^{\tilde{\tau}}$ and $W$ is open in $H]$, then $\tau$ is a locally compact group topology of $H$, and the map $\pi$ induces an open continuous homomorphism of $H^{\tau}$ onto $(H / L)^{\bar{\tau}}$. For each $f \in L^{1}\left((H / L)^{\tau}\right)$, put $h^{\prime}(f)=f \circ \pi$, then $h^{\prime}(f)$ belongs to $L^{1}\left(H^{r}\right)$ and $h^{\prime} h=h$. Thus we have $h\left(L^{1}(G)\right)=h^{\prime} h\left(L^{1}(G)\right) \subset L^{1}\left(H^{\tau}\right)$.

Finally we prove the general case. Let $\Lambda$ be the closure of $\alpha(Y)$ in $\hat{G}$. Then there exists a homomorphism $h^{\prime \prime}$ of $L^{1}(G / K)$ into $M(H)$ with the dual map $(Y, \alpha)$, where $K$ is the annihilator of $\Lambda$ in $G$. Since $A(\Lambda)$ coincides with the set $\left[\left.\hat{f}\right|_{\Lambda}: f \in A(\hat{G})\right]$, we can reduce the problem to the preceding case; thus we have $h\left(L^{1}(G)\right)=h^{\prime \prime}\left(L^{1}(G / K)\right) \subset L^{1}\left(H^{T}\right)$ for some $\tau \in \mathfrak{I}(H)$. This completes the proof.

LEMMA 2. If $Y$ is an open coset and $\alpha$ is an affine map, then we get the same conclusion as Lemma 1.

Proof. Let $r_{2}$ be an element of $H$ such that $Y-r_{2}$ is an open subgroup of $\hat{H}$. There exist a continuous homomorphism $\beta$ of $Y-r_{2}$ into $\hat{G}$ and $r_{1} \in \hat{G}$ such that

$$
\alpha(r)=\beta\left(r-r_{2}\right)-r_{1} \quad(r \in Y) .
$$


By Lemma 1, there exist an element $\tau \in \mathfrak{I}(H)$ and a continuous homomorphism $h^{\prime}$ of $L^{1}(G)$ into $L^{1}\left(H^{T}\right)$ with the dual map $\left(Y-r_{2}, \beta\right)$. If we define $h_{1}$ and $h_{2}$ by

$$
h_{1}(f)=r_{1} f \quad\left(f \in L^{1}(G)\right) ; \quad h_{2}(g)=r_{2} g \quad\left(g \in L^{1}\left(H^{\tau}\right)\right),
$$

then $h_{1}$ and $h_{2}$ are homomorphisms of $L^{1}(G)$ into $L^{1}(G)$ and $L^{1}\left(H^{r}\right)$ into $L^{1}\left(H^{r}\right)$, respectively. Since $h=h_{2} h^{\prime} h_{1}$, the range of $h$ is contained in $L^{1}\left(H^{r}\right)$ and Lemma 2 is proved.

Let $J(H)$ be the set of all the idempotent measures in $M(H)$, and for each $\mu \in J(H)$ we put $S(\mu)=[r \in \hat{H}: \hat{\mu}(r)=1]$.

Lemma 3. If $\mu$ is an element of $J(H)$, then there exist finitely many compact subgroups $K_{1}, K_{2}, \cdots, K_{n}$ of $H$ such that

(i) $m_{K_{i}}$ and $m_{K_{j}}$ are mutually singular for $i \neq j$,

(ii) for $i$ and $j$, we have $m_{K_{i}} * m_{K_{j}}=m_{K_{i}+K_{j}} \ll m_{K_{l}}$ (absolutely continuous with respect to $m_{K_{l}}$ ) for some $l$,

(iii) $\mu \ll \sum_{i=1}^{n} m_{K_{i}}$,

where $m_{K}$ denotes the Haar measure of a compact group $K$.

Proof. There exists a set $\left[K_{1}, K_{2}, \cdots, K_{m}\right]$ of finitely many compact subgroups of $H$ which satisfies the conditions (i) and (ii) (cf. [5]). We can choose finitely many compact subgroups $K_{m+1}, \cdots, K_{n}$ of $H$ (if necessary) so that $\left[K_{1}, K_{2}, \cdots, K_{n}\right]$ satisfies the conditions (i), (ii) and (iii), and this completes the proof.

Lemma 4. If there exist an open coset $\Lambda$ and an affine map $\bar{\alpha}$ of $\Lambda$ into $\hat{G}$ such that $Y \subset \Lambda,\left.\bar{\alpha}\right|_{Y}=\alpha$, then we get the conclusion of the theorem.

Proof. Since $Y$ is an element of the coset ring of $\hat{H}$, there exists $\mu \in J(H)$ such that $S(\mu)=Y$. Since $\mu$ is determined by $h$, we express $\mu$ by $j(h)$. Let $\left[K_{1}, K_{2}, \cdots, K_{n}\right]$ be a set of finitely many compact subgroups of $H$ which satisfies (i), (ii) and (iii) of Lemma 3. We decompose $\mu$ as $\mu=\lambda_{1}+\lambda_{2}+\cdots+\lambda_{n}, \lambda_{i} \ll m_{K_{i}}(i=1,2, \cdots, n)$, and we proceed by induction on the number $n$ of $\left[K_{1}, K_{2}, \cdots, K_{n}\right]$. Thus we suppose that Lemma 4 is true if $n \leqq k$, and prove that Lemma 4 is also true for $n=k+1$.

We can suppose without loss of generality that $K_{n}$ is minimal in the sense that $K_{i} / K_{n} \cap K_{i}$ is infinite for $i \neq n$. Then since $\mu=\mu * \mu=\lambda_{n} * \lambda_{n}+$ $\sum_{i \neq n \text { or } j \neq n} \lambda_{i} * \lambda_{j}$, we get $\sum_{i \neq n \text { or } j \neq n} \lambda_{i} * \lambda_{j} \ll \sum_{i=1}^{n-1} m_{K_{i}}$ and $\lambda_{n} \in J(H)$. If we put

$$
\begin{aligned}
& h_{1}: f \in L^{1}(G) \mapsto h(f) * \lambda_{n} * \mu \in M(H), \\
& h_{2}: f \in L^{1}(G) \mapsto h(f) *\left(\mu-\mu * \lambda_{n}\right) \in M(H),
\end{aligned}
$$


then $h_{1}$ and $h_{2}$ are homomorphisms which satisfy $h_{1}(f)+h_{2}(f)=h(f)$ ( $f \in L^{1}(G)$ ). Since $\left[K_{1}, K_{2}, \cdots, K_{n-1}\right]$ satisfies the conditions (i), (ii) and (iii) of Lemma 3 for $\mu=j\left(h_{2}\right)$, we have by the assumption of the induction that $h_{2}\left(L^{1}(G)\right) \subset \sum_{r \in A} L^{1}\left(H^{r}\right)$ for some finite subset $A \subset \mathfrak{I}(H)$, and we have only to prove the lemma for $h=h_{1}$. Therefore we can assume here without loss of generality that $\lambda_{n} * \mu=\mu$, that is $S\left(\lambda_{n}\right) \supset S(\mu)$. Obviously, $\lambda_{n}$ is an irreducible idempotent, and hence there exist $r_{1}, r_{2}, \cdots, r_{m} \in \hat{H}$ such that $d \lambda_{n}=\left[\left(x, r_{1}\right)+\cdots+\left(x, r_{m}\right)\right] d m_{K_{n}}$, where $r_{i}-r_{j}(i \neq j)$ does not belong to the annihilator of $K_{n}$.

For each $i$, let $\sigma_{i}$ be an element of $J(H)$ such that $d \sigma_{i}=\left(x, r_{i}\right) d m_{K_{n}}$ and let $h_{i}$ be a homomorphism of $L^{1}(G)$ into $M(H)$ with the dual map $\left(S\left(\sigma_{i}\right) \cap \Lambda,\left.\bar{\alpha}\right|_{S\left(\sigma_{i}\right) \cap \Lambda}\right)$. Let $h_{i}^{\prime}$ and $h_{i}^{\prime \prime}$ be homomorphisms of $L^{1}(G)$ into $M(H)$ such that $h_{i}^{\prime}(f)=h(f) * \sigma_{i}$ and $h_{i}^{\prime \prime}(f)=h_{i}(f) *\left(\sigma_{i}-\sigma_{i} * \mu\right)$, and then we have $h_{i}^{\prime}(f)=h_{i}(f)-h_{i}^{\prime \prime}(f)\left(f \in L^{1}(G)\right)$. By Lemma $2, h_{i}$ maps $L^{1}(G)$ into $L^{1}\left(H^{\tau_{i}}\right)$ for some $\tau_{i} \in \mathfrak{I}(H)$, and since $j\left(h_{i}^{\prime \prime}\right)$ is absolutely continuous with respect to $\sum_{j=1}^{n-1} m_{K_{j}}$ we have again by the assumption of the induction that $h_{i}^{\prime \prime}$ maps $L^{1}(G)$ into $\sum_{r \in B_{i}} L^{1}\left(H^{r}\right)$ for some finite subset $B_{i} \subset \mathfrak{I}(H)$, and consequently we get

$$
h\left(L^{1}(G)\right) \subset \sum_{i=1}^{m} h_{i}\left(L^{1}(G)\right)-\sum_{i=1}^{m} h_{i}^{\prime \prime}\left(L^{1}(G)\right) \subset \sum_{\tau \in\left(\cup_{i=1}^{m}\right.} \sum_{\left.B_{i}\right) \cup\left\{\tau_{1}, \cdots, \tau m\right\}} L^{1}\left(H^{\tau}\right),
$$

and this completes the proof.

THE PROOF OF THE THEOREM. Let $(Y, \alpha)$ be the dual map of $h$. There exist a set of pairwise disjoint elements $\left\{Y_{i}\right\}_{i=1}^{n}$ of the coset ring of $\hat{H}$, a. set of open cosets $\left\{K_{i}\right\}_{i=1}^{n}$ of $\hat{H}$ and a set of affine maps $\left\{\alpha_{i}: K_{i} \rightarrow \hat{G}\right\}_{i=1}^{n}$ such that

$$
Y=Y_{1} \cup \cdots \cup Y_{n}, \quad K_{i} \supset Y_{i},\left.\quad \alpha\right|_{Y_{i}}=\left.\alpha_{i}\right|_{Y_{i}} \quad(i=1,2, \cdots, n) .
$$

If we denote by $h_{i}$ a homomorphism of $L^{1}(G)$ into $M(H)$ with the dual $\operatorname{map}\left(Y_{i},\left.\alpha\right|_{Y_{i}}\right)(i=1,2, \cdots, n)$, then we have $h(f)=h_{1}(f)+\cdots+h_{n}(f)$ $\left(f \in L^{1}(G)\right)$. By Lemma 4 we have $h_{i}(f) \in \sum_{\tau \in A_{i}} L^{1}\left(H^{r}\right)$ for some finite subset $A_{i} \subset \mathfrak{I}(H)$, and hence $h(f)$ belongs to $\sum_{\tau \in} \cup_{i=1}^{n} A_{i} L^{1}\left(H^{r}\right)$ for each $f \in L^{1}(G)$ and $i$, and thus the theorem is proved.

REMARK. If we refer to [4], we can see that $\sum_{\tau \in \mathfrak{I}(H)} L^{1}\left(H^{\tau}\right)$ is a subalgebra of $M(H)$ and that the norm closure of $\sum_{r \in \mathfrak{T}(H)} L^{1}\left(H^{r}\right)$ in $M(H)$ is a proper closed subalgebra of $M(H)$ if $H$ is not discrete. This means that the set of the elements of the form $h(x)(x \in G)$, where a LCA group $G$ and a homomorphism $h$ of $L^{1}(G)$ into $M(H)$ vary arbitrarily, constitutes the subalgebra $\sum_{\tau \in \mathfrak{I}(H)} L^{1}\left(H^{r}\right)$ contained (if $H$ is not discrete) in a proper closed subalgebra of $M(H)$. 


\section{REFERENCES}

1. P. J. Cohen, On a conjecture of Littlewood and idempotent measures, Amer. J. Math. 82 (1960), 191-212. MR 24 \#A3231.

2. - On homomorphisms of group algebras, Amer. J. Math. 82 (1960), 213-226. MR 24 \#A3232.

3. P. Eymard, Homomorphismes des algèbres de groupe, Séminaire Bourbaki, 1961/62, $\mathrm{n}^{0} 231$.

4. J. Inoue, Some closed subalgebras of measure algebras and a generalization of P. J. Cohen's theorem, J. Math. Soc. Japan 23 (1971), 278-294. MR 44 \#7223.

5. T. Ito and I. Amemiya, A simple proof of the theorem of P. J. Cohen, Bull. Amer. Math. Soc. 70 (1964), 774-776. MR 29 \#4862.

6. W. Rudin, Fourier analysis on groups, Interscience Tracts in Pure and Appl. Math., no. 12, Interscience, New York, 1962. MR 27 \#2808.

Department of Mathematics, Nagoya Institute of Technology, Nagoya, JAPAN 